\title{
Valsartan inhibits RhoA-ROCK2-MYL pathway in rat model of alcoholic cardiomyopathy
}

\author{
LUYIFEI LI ${ }^{1}$, LING JING ${ }^{2}$, JIYI ZHAO ${ }^{2}$, JIACHEN LV $^{3}$, WEN YANG ${ }^{4}$, WEIMIN LI ${ }^{5}$ and LIJUN ZHOU ${ }^{2}$ \\ Departments of ${ }^{1}$ Internal Critical Illness and ${ }^{2}$ The Fourth Cardiovascular, \\ The First Affiliated Hospital of Harbin Medical University; ${ }^{3}$ Department of Colorectal Surgery, \\ The Tumor Hospital of Harbin Medical University; Departments of ${ }^{4}$ The First Nephrology and \\ ${ }^{5}$ The Fifth Cardiovascular, The First Affiliated Hospital of Harbin Medical University, Harbin, Heilongjiang 150000, P.R. China
}

Received March 14, 2018; Accepted November 1, 2018

DOI: $10.3892 /$ etm.2019.8079

\begin{abstract}
The present study aimed to investigate variations in theRashomologgenefamily,memberA(RhoA)-Rho-associated protein kinase 2 (ROCK2)-myosin light chain (MYL) pathway in a rat model of alcoholic cardiomyopathy (ACM) and the role of angiotensin-converting enzyme inhibitor drugs. Rat models of ACM were established via alcoholic gavage + free access to alcohol. The structural and functional changes of the heart were analyzed by hematoxylin-eosin staining, Masson's trichrome staining, immunohistochemistry staining, western blotting and fluorescence quantitative polymerase chain reaction. A total of 16 weeks later, a decreased ejection fraction and left ventricular fractional shortening in the alcohol group compared with the control group were demonstrated resulting in an increased left ventricular end diastolic diameter. These adverse effects were ameliorated following treatment with valsartan. In addition, the alcohol group revealed a disorganized arrangement of myocardial filaments, which was improved upon treatment with valsartan. RhoA and ROCK 2 protein expression significantly increased in myocardial cells in the alcohol compared with the control group. Following drug intervention with valsartan, expression of RhoA and ROCK2 proteins were inhibited in the alcohol group. Furthermore, significantly elevated RhoA and ROCK2 and decreased MYL protein and mRNA expression in the
\end{abstract}

Correspondence to: Dr Weimin Li, Department of The Fifth Cardiovascular, The First Affiliated Hospital of Harbin Medical University, 23 Post Street, Nangang, Harbin, Heilongjiang 150000, P.R. China

E-mail: liweimin_2009@163.com

Dr Lijun Zhou, Department of The Fourth Cardiovascular, The First Affiliated Hospital of Harbin Medical University, 23 Post Street, Nangang, Harbin, Heilongjiang 150000, P.R. China

E-mail: doczlj@126.com

Key words: alcoholic cardiomyopathy, valsartan, Ras homolog gene family, member A, Rho-associated protein kinase, myosin light chain alcohol group was demonstrated compared with the control group. Administration of valsartan reversed the expression profile of RhoA, ROCK and MYL in ACM. Expression of RhoA and ROCK were elevated with downregulation of MYL resulting in heart failure. However, the angiotensin receptor antagonist diminished the expression of RhoA and ROCK and enhanced the expression of MYL. The results of the present study suggest a curative effect of valsartan in ACM.

\section{Introduction}

Long-term alcohol consumption frequently leads to development and progression of non-ischemic dilated cardiomyopathy (NIDCM), also known as alcoholic cardiomyopathy (ACM) (1). Alcohol exerts diverse toxic effects on the heart contributing to heart failure, conduction block, atrial fibrillation, myocardial remodeling and cardiac anomalies associated with metabolism and function. In NIDCM patients, who never stop their alcohol intake, the 4-year mortality rate was as high as $50 \%(2,3)$. However, the mechanism of action of alcohol in NIDCM has not been elucidated.

Alterations in the metabolism of fatty acid ethyl esters cause decreased $\beta$-oxidation of fatty acids and contribute to metabolic disturbances in myocardial cells (4-6). Previous studies suggest alcohol intake as a cause of increased plasma homocysteine, which is associated with oxidative stress, mitochondrial dysfunction and inflammation, all of which induce myocardial fibrosis and cardiac remodeling (7-9). Tenascin, a major protein of the extracellular matrix is divided into 6 subtypes, produced by fibroblasts, along with collagen mediates the process of fibrosis (10). Peroxisome proliferator-activated receptor $\alpha$ $(\operatorname{PPAR} \alpha)$ is a key enzyme involved in the regulation of fatty acid oxidation $(11,12)$. Retinoid $x$ receptor $\alpha(\mathrm{RXR} \alpha)$ PPAR $\alpha$ and $\mathrm{RXR} \alpha$ are the major nuclear transcription factors involved in the energy metabolism of fatty acid in myocardial cells and in remodeling the myocardium (13). Angiotensin II via activation of angiotensin II type I receptor increases superoxide anion generated by NADPH, while suppressing angiotensin II ameliorates oxidative stress and fibrosis (14). Almost all cases of ACM are associated with cardiac remodeling induced by myocardial fibrosis and oxidative stress (14). Nevertheless, the mechanisms of ACM remain unclear. 
Several hypotheses have been postulated regarding the pathogenesis of ACM, including the toxic effects of alcohol on the heart and enhanced oxidative stress (15). However, only limited studies have focused on the effect of Ras homolog gene family, member A (RhoA), Rho-associated protein kinase 2 (ROCK2) and myosin light chain (MYL) in the pathogenesis of ACM. A previous study has indicated that ethanol could disrupt the junction between intestinal epithelial cells through activation of the RhoA-ROCK pathway (16). The RhoA-ROCK pathway alters the smooth muscle cell cytoskeleton and causes remodeling of the respiratory tract in infant mice (17). In nucleus pulposus cells, renin activates the RhoA-ROCK pathway, thereby inducing the remodeling of the cytoskeleton (18). The RhoA/Rho-kinase pathway serves an important role in various fundamental cellular functions, including production of excessive reactive oxygen species, leading to the development of cardiovascular diseases (19). Rho-kinase also upregulates $\mathrm{NAD}(\mathrm{P}) \mathrm{H}$ oxidases (Nox1, Nox4, gp91phox and p22phox), and augments AngII-induced ROS production $(20,21)$. The role of RhoA-ROCK in the pathogenesis of ACM is still not clearly elucidated. The present study aims to interpret altered expression of the RhoA-ROCK pathway, MYL and its downstream targets in the pathogenesis, and treatment of ACM. In addition, the therapeutic effects of valsartan on ACM were analyzed. Future research aimed at elucidating the pathogenesis of ACM may contribute to significant breakthroughs that might prove beneficial for the diagnosis and treatment of ACM.

\section{Materials and methods}

Instruments and reagents. Refrigerators and deep freezers $\left(4^{\circ} \mathrm{C},-20^{\circ} \mathrm{C}\right.$ and $\left.-80^{\circ} \mathrm{C}\right)$ (Haier, Qingdao, China); light microscopes (Olympus Corporation, Tokyo, Japan); color Doppler ultrasound diagnostic system (GE Healthcare, Chicago, IL, USA); pathological image analysis system (Motic Images Advanced 3.0; Motic Asia, Hong Kong, China); gel-image analyzer (Bio-Rad Laboratories, Inc., Hercules, CA, USA); electronic scale (Shanghai Scale, Shanghai, China); liquid nitrogen biological container (Chengdu Jinfeng Liquid Nitrogen Container Co., Ltd., Chengdu, China); Langendorff perfusion system (Etiological Lab of Harbin Medical University, Harbin, China); microplate reader (Tekon Scientific Corp., Taipei city, Taiwan); electrophoresis system and electronic transfer (Beijing Liuyi Biotechnology Co., Ltd., Beijing, China); centrifuge (Kaidi Machinery Co., Ltd., Jiangsu, China); quantitative PCR system (Shanghai Zhiyan, China); thermostatic water (Shanghai Medical Analytic Instrument Factory, Shanghai, China) were used in the present study.

In addition the following reagents were purchased: Valsartan capsules (7 tablets, $80 \mathrm{mg} /$ tablet; Novartis International AG, Basel, Switzerland); $98 \%$ ethanol (500 ml), $10 \%$ chloral hydrate, heparin, $\mathrm{Ca}^{2+}$-free Tyrode solution, $\mathrm{Ca}^{2+}$-contained Tyrode solution and PBS solution $(8.0 \mathrm{~g}$ of $\mathrm{NaCl}, 0.2 \mathrm{~g}$ of $\mathrm{KCl}, 1.26 \mathrm{~g}$ of $\mathrm{Na}_{2} \mathrm{HPO}_{4} \cdot 12 \mathrm{H}_{2} \mathrm{O}$, and $0.2 \mathrm{~g}$ of $\mathrm{KH}_{2} \mathrm{PO}_{4}$ adjust the $\mathrm{pH}$ to 7.2 with $1 \mathrm{~mol} / \mathrm{l} \mathrm{HCl}$ or $1 \mathrm{~mol} / \mathrm{l}$ $\mathrm{NaOH}$ to $1,000 \mathrm{ml}$, PBS was provided by the Etiological Lab of Harbin Medical University), collagenase II and albumin (Zhongtian World, Harbin, China); radioimmunoprecipitation assay lysis buffer, Benzonase, TEMED, bicinchoninic acid kit, 10\% SDS, 30\% Acr-Bis (29:1), Tris, SDS buffer, enhanced chemiluminescence reagent substrate (cat. no. no32106; Invitrogen; Thermo Fisher Scientific, Inc., Waltham, MA, USA ), skimmed milk powder (Beyotime Institute of Biotechnology, Haimen, China); polyvinylidene difluoride (PVDF) membrane (EMD Millipore, Billerica, MA, USA); mouse anti-RhoA polyclonal antibody $(1: 1,000$; cat. no. ab54835; Abcam, Cambridge, MA, USA), mouse anti-MYL1 polyclonal antibody (1:1,000; cat. no. PA5-29635 Invitrogen; Thermo Fisher Scientific, Inc.), goat anti-ROCK polyclonal antibody $(1: 1,000$; sc-1851; Santa Cruz Biotechnology, Inc., Dallas, TX, USA); $\beta$-actin (1:5,000; cat. no. ab8227; Abcam) horseradish peroxidase (HRP)-labeled mouse anti-immunoglobulin (Ig)G antibody (1:5,000; cat. no. sc-2005; Santa Cruz Biotechnology, Inc., Dallas, TX, USA), HRP-labeled goat anti-IgG antibody (1:5,000; cat. ab6721; Abcam); citrate sodium buffer, PBST, $30 \% \mathrm{H}_{2} \mathrm{O}_{2}$, and hematoxylin (provided by Etiological Lab of Harbin Medical University); RNA extraction kit Trizol (Invitrogen; Thermo Fisher Scientific, Inc., Waltham, MA, USA); Accupower RocketScript RT PreMix (Bioneer Corporation, Daejeon, Korea); Real MasterMix (SYBR Green, Tiangen, China); primer synthesis for $\mathrm{qPCR}$ (Bioneer Corporation).

Subjects. A total 120, 8-10 weeks (280-300 g) healthy male Wistar rats were purchased from the Changchun Yisi Experimental Animal Co., Ltd., (Changchun, China). Animals were maintained in a controlled environment (12-h light/dark cycle; temperature, $27 \pm 2^{\circ} \mathrm{C}$; humidity, $35 \pm 5 \%$ ). The animals were fed a standard pellet diet and water was freely available. Animals were maintained at the Experimental Animal Center of the First Affiliated Hospital of Harbin Medical University. This study was approved by the First Affiliated Hospital of Harbin Medical University.

Establishing a rat model of ACM. A total of 120 male Wistar rats were randomly divided into three groups, $n=40$ namely, the control group, the alcohol group and the alcohol + valsartan group (treatment group). ACM was induced in rats through alcoholic gavage and free access to alcohol. During the first week, rats received $60 \%$ ethanol $(6 \mathrm{ml} / \mathrm{kg} /$ day $)$ through gavage and had free access to $10 \%$ ethanol all day long. In the second week, they received $12 \mathrm{ml} / \mathrm{kg} /$ day ethanol and had free access to $10 \%$ ethanol. Through the third week, rats were continued to receive $60 \%$ ethanol at a dose of $12 \mathrm{ml} / \mathrm{kg}$ /day but had free access to $20 \%$ ethanol. From the 4th to 16 th week, rats were received a gavage of $60 \%$ ethanol at a dose of $15 \mathrm{ml} / \mathrm{kg} / \mathrm{day}$, which was carried out twice per day, along with free access to $20 \%$ ethanol. For rats in the treatment group, valsartan at a dose of $8 \mathrm{mg} / \mathrm{kg} /$ day was additionally administered. Rats in the control group were fed with regular water and food. The experimental design is presented in Fig. 1.

Doppler echocardiography. Rats were anesthetized by intraperitoneal (IP) injection of $10 \%$ chloral hydrate $(300 \mathrm{mg} / \mathrm{kg})$. Cardiac color ultrasonic scanner (GE Healthcare) and a probe $(10 \mathrm{MHz})$ was used to examine the variations in the structure and function by professional sonographers. The following were examined, namely, the left ventricular end diastolic diameter (LVDD), ejection fraction (EF) of the left ventricle, 
left ventricular fractional shortening (FS) and the early/atrial ratio in three consecutive cardiac cycles. The results were averaged.

Collection of specimen. The animals were euthanized under sodium pentobarbital anesthesia. After sacrifice the rat hearts were isolated and rinsed with pre-cooled normal saline. Tissue specimens were collected from the transverse section of the left ventricular myocardium. Briefly, tissues were cut from the apex of the heart and isolated from the free wall of the left ventricle parallel to its longitude axis on ice. Specimens were fixed in $4 \%$ formaldehyde for $4 \mathrm{~h}$ at $4^{\circ} \mathrm{C}$, were paraffin embedded, serial sectioned and stained with hematoxylin for 5-10 $\mathrm{min}$ at room temperature. Remaining tissues were preserved at $-80^{\circ} \mathrm{C}$ until further use.

Hematoxylin \& eosin $(H \& E)$ staining. Sections were dewaxed twice in xylene (10 min each). Sections were rehydrated sequentially in descending series of alcohol for 5 min each in anhydrous, 90, 80 and 70\% alcohol. Sections were then treated with phosphate buffered saline, $0.1 \%$ Tween-20 (PBST) for 2 min. Specimens were stained by immersing in hematoxylin for 5-10 min at room temperature, treated with $1 \%$ acid alcohol for $3 \mathrm{sec}$, washed with running water for $10 \mathrm{~min}$, washed with distilled water for 1 or $2 \mathrm{~min}$, staining with $0.5 \%$ eosin for 1-3 min and washed with distilled water for $2 \mathrm{sec}$. Specimens were then dehydrated twice in $95 \%$ ethanol for 2 min each and cleared by treating twice with xylene for $5 \mathrm{~min}$ each. Sections were then mounted with neutral balsam and observed under a light microscope. As anticipated, the nuclei were stained red, while the cytoplasm was stained pink.

Masson's trichrome staining. Sequentially, specimens were dewaxed, washed with running water and treated with a mordant for $30 \mathrm{~min}$. Specimens were then stained with hematoxylin for $20 \mathrm{~min}$ at room temperature, washed with running water, treated with acidic alcohol for 10 to $15 \mathrm{sec}$, washed again with running water, treated with ammonia for 10 to $15 \mathrm{sec}$ and the reaction was terminated by washing with running water. Thereafter, specimens were stained in Masson solution for $1 \mathrm{~min}$ at room temperature, washed in acetic acid and observed under a light microscope.

Immunohistochemistry(IHC). Paraffin sections were dewaxed, incubated with $3 \% \mathrm{H}_{2} \mathrm{O}_{2}$ for 5 to $10 \mathrm{~min}$ at $25^{\circ} \mathrm{C}$ to block endogenous peroxidase activity, rinsed with distilled water and treated twice with PBS (5 min each). Sections (4-6 $\mu$ m-thick) were then blocked at at room temperature in 5 to $10 \%$ normal goat serum diluted in PBS for $10 \mathrm{~min}$ and incubated overnight with primary antibodies at $37^{\circ} \mathrm{C}$ for 1 to $2 \mathrm{~h}$ or $4^{\circ} \mathrm{C}$ overnight. Sections were washed thrice in PBS ( 5 min each), incubated with biotin-labeled secondary antibodies at $37^{\circ} \mathrm{C}$ for 10 to $30 \mathrm{~min}$, washed thrice in PBS (5 min each), incubated with HRP- or alkaline phosphatase-labeled streptavidin for 10 to $30 \mathrm{~min}$ at $37^{\circ} \mathrm{C}$ and washed thrice with PBS (5 min each). After washing, slides were incubated with 3,3'-diaminobenzidine tetrahydrochloride (Sigma-Aldrich; Merck KGaA, Darmstadt, Germany) for $2 \mathrm{~h}$ at room temperature and immediately washed under tap water following color development. Slides were then counter stained with hematoxylin for $10 \mathrm{~min}$ at room temperature. Slides were mounted with dibutyl phthalate xylene and observed under a light microscope (Carl Zeiss AG, Oberkochen, Germany).

Sample extraction. In a water bath set at $39^{\circ} \mathrm{C}$, the Langendorff channel was rinsed twice with deionized water and filled with calcium-free tyrode solution. Digestive solution was prepared using $8 \mathrm{mg}$ albumin, $0.5 \mathrm{mg}$ collagenase and $50 \mathrm{ml}$ calcium-free Tyrode solution. Hank's Balanced Salt Solution stored at $-20^{\circ} \mathrm{C}$ was thawed for later use.

Extraction of myocardial cells. Rats represented as subjects were IP injected with $2 \mathrm{ml}$ of heparin. Rats were anesthetized $20 \mathrm{~min}$ later by IP injection of $2 \mathrm{ml}$ chloral hydrate (10\%). Thoracic surgery was conducted on the rats to isolate the heart. The heart was isolated by excising the aorta at the distal end. The heart was immediately transferred into a calcium-containing Tyrode solution in a culture dish, where the surrounding pulmonary tissues and vessels were dissected rapidly. The aorta has three branches at the upper end and is located beneath the two white thymus glands. At the bifurcation, the aorta was dissected to expose its outlet into which the $12^{\#}$ needle of the $20 \mathrm{ml}$ injector containing calcium-free Tyrode solution was inserted, followed by ligation with a suture for fixation. The injector was slowly pushed and the needle connected with the heart was inserted in the T-Cock. Thereafter, perfusion with calcium-free Tyrode solution was carried out. When the level of the solution decreased beneath the neck of the tube, digestive solution was placed in the Langendorff device. The above procedures were repeated and the digestive solution was collected in a beaker. Digestion was carried out for 30 min with continuous supplementation of digestive solution. After digestion, the white and widened heart tissue was placed in a culture dish supplemented with $\mathrm{KB}$ solution. Following excising the atrium and auriculars, the remaining tissue was dissected and cut into pieces, placed in a centrifuge tube supplemented with the KB solution, and beaten with a pipette. A drop of myocardial cell suspension was dripped onto a glass slide and observed under a light microscope (Carl Zeiss AG) for the estimation of survival rate. Samples were immediately centrifuged $2,000 \mathrm{xg}$ for $10 \mathrm{~min}$ at $4^{\circ} \mathrm{C}$ and stored at $-80^{\circ} \mathrm{C}$ until further use.

Estimation of protein concentration in samples. Cell lysis was carried out on ice using PMSF (100:1). Cell lysates were mixed well by beating and vibration. After $1 \mathrm{~min}$ of vibration, lysates were placed for $5 \mathrm{~min}$, centrifuged at $1,000 \mathrm{x} \mathrm{g}$ for $10 \mathrm{~min}$ at $4^{\circ} \mathrm{C}$ and the supernatants were collected for protein quantification. In a 96-well plate, samples were diluted in deionized water to a volume of $20 \mu \mathrm{l}$. A total of $200 \mu \mathrm{l}$ of solution A and B (50:1) was added to each well and incubated for $30 \mathrm{~min}$. Samples were mixed with the loading buffer (1:4) and denatured for $10 \mathrm{~min}$. A microplate reader was used to determine the concentration of protein in the samples.

RNA extraction and reverse transcription-quantitative polymerase chain reaction (RT-qPCR). RNA from myocardial tissue was extracted using TRIzol ${ }^{\circledR}$ reagent (Invitrogen; Thermo Fisher Scientific, Inc.) as previously described. cDNA synthesis was performed using an RT kit (cat. no. FSQ-101; Toyobo Life Science, Osaka, Japan), according to the manufacturer's 


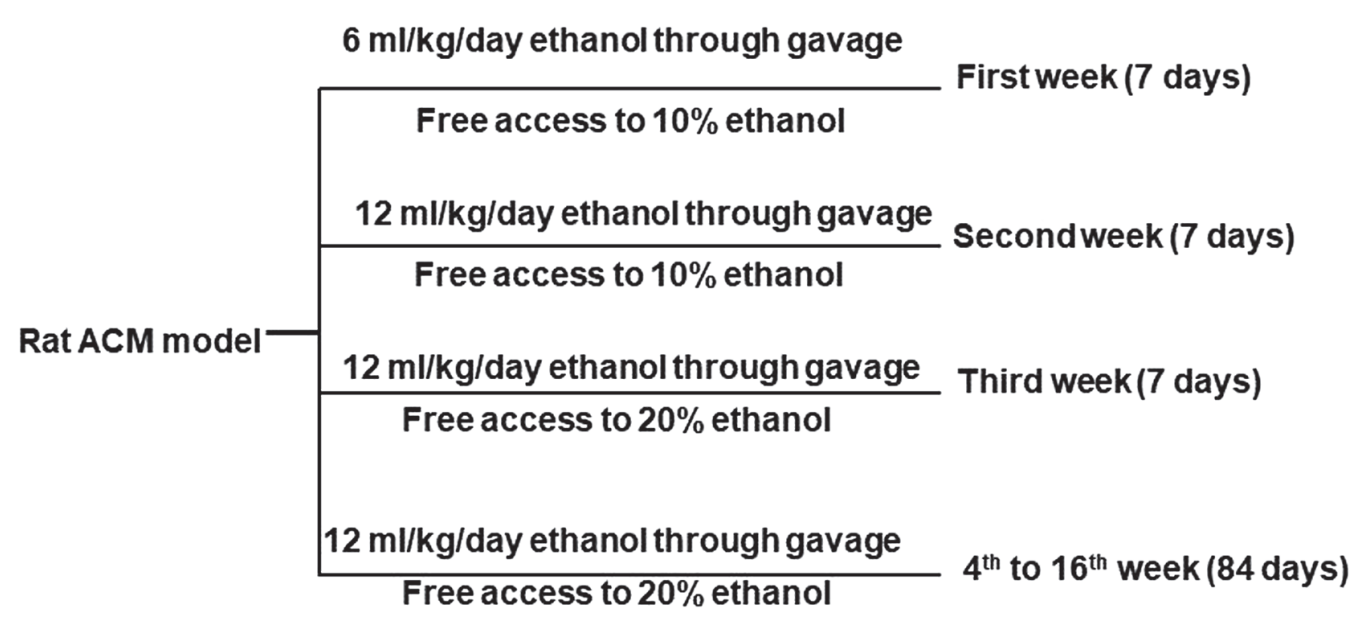

Figure 1. The diagram of experimental design. ACM, alcoholic cardiomyopathy.

protocol. qPCR was conducted using the LightCycler ${ }^{\circledR}$ qPCR apparatus (Roche Molecular Systems, Inc.). The following thermocycling conditions were used: 40 cycles of $95^{\circ} \mathrm{C}$ for $15 \mathrm{sec}$ and $60^{\circ} \mathrm{C}$ for $60 \mathrm{sec}$ with Fast Start SYBR Green master mix (Roche Molecular Systems, Inc.). mRNA expression results were analyzed using the $2^{-\Delta \Delta C q}$ method (22). The following primer sequences were used: RhoA forward, $5^{\prime}$-CTC TCTTATCCAGACACCGATGT-3' and reverse, 5'-TGTGCT CGTCATTCCGAAGG-3'; ROCK forward, 5'-GTTCGTCAT AAGGCATCACAGA-3' and reverse, 5'-TGTTGGCAAAGG CCATAATATCT-3'; MYL forward, 5'-CCCGAAGGGCTT TCACAATCT-3' and reverse, 5'-CCCACTCTTCCAAAC AGCAG-3'.

Western blotting. The composition of the lower gel included, $10 \mathrm{ml}$ water $\left(\mathrm{H}_{2} \mathrm{O}\right), 3.3 \mathrm{ml} 30 \%$ Arc-Bis (29:1), $3.8 \mathrm{ml} 1 \mathrm{M}$ Tris $(\mathrm{pH}=8.8), 0.1 \mathrm{ml} 10 \%$ SDS, $0.1 \mathrm{ml} \mathrm{10 \%}$ ammonium persulfate and $0.004 \mathrm{ml}$ tetramethylethylenediamine (TEMED). The composition of the upper gel included, $3.4 \mathrm{ml} \mathrm{H}_{2} \mathrm{O}, 0.85 \mathrm{ml} 30 \%$ Arc-Bis (29:1), $0.625 \mathrm{ml} 1 \mathrm{M}$ Tris (pH=6.8), $0.05 \mathrm{ml} 10 \%$ SDS, $0.05 \mathrm{ml} 10 \%$ ammonium persulfate and $0.005 \mathrm{ml}$ TEMED.

In the plate, the lower gel and upper gel were sequentially added without air bubbles with the immediate insertion of the comb in the upper gel. After coagulation, electrophoresis buffer was added into the electrophoresis apparatus and the comb was removed. After loading the proteins $(25 \mu \mathrm{g})$ and marker, electrophoresis was carried out at $80 \mathrm{~V}$ for $40 \mathrm{~min}$ and later at $120 \mathrm{~V}$ until the proteins and marker reached the bottom of the plate. For blotting, the following were placed in a sequential order in the electrophoretic unit: Sponge pad, filter paper, gel, PVDF membrane, filter paper and sponge pad from the negative electrode to the positive electrode without air bubbles. In the transfer apparatus, transfer buffer was added and the transfer was carried out at a constant current of $200 \mathrm{~mA}$ for $3 \mathrm{~h}$ at $4^{\circ} \mathrm{C}$. Membranes were blocked in 5\% skimmed milk on a shaker for $2 \mathrm{~h}$ at room temperature. Membranes were then incubated with RhoA anti-mouse polyclonal antibodies (1:200), MYL anti-mouse polyclonal antibody (1:500), ROCK anti-goat polyclonal antibody (1:200) and $\beta$-actin (1:500) overnight at $4^{\circ} \mathrm{C}$, washed thrice with TBST (5 min each), incubated with the corresponding HRP-conjugated secondary antibodies (cat. nos. ab7061, ab7125 and ab97085; all Abcam) for $1 \mathrm{~h}$ at room temperature, and washed thrice with TBST. After exposure in the dark with enhanced chemiluminescence reagent (Beyotime Institute of Biotechnology; cat. no. P0018M), membranes were scanned in the gel imaging system (cat. no. 4466613; E-Gel ${ }^{\mathrm{TM}}$ Imager System with E-Gel ${ }^{\mathrm{TM}}$ Adaptor and Bio-Rad: Universal Hood II ) and densitometry was performed using Image Lab software (version 2.0.1; Bio-Rad Laboratories, Inc.).

Statistical analysis. All statistical analyses were performed using SPSS 18.0 software (SPSS, Inc., Chicago, IL, USA). Results were expressed as the mean \pm standard deviation. Experiments were repeated for at least 3 times. The independent sample t-test was used for comparison between two groups. One-way analysis of variance followed by Tukey's post-hoc test was applied for comparison among groups; $\mathrm{P}<0.05$ were considered to indicate a statistically significant difference.

\section{Results}

Verification of non-ischemic dilated cardiomyopathy model. In the alcohol group, ST-segment elevation (>1/2 R waves) in left ventricular coronary arteries following left coronary artery ligation demonstrated a single-peak curve, which is a sign of successful rat non-ischemic dilated cardiomyopathy (Fig. 2A). Ultrasound detection demonstrated that the thickness of the left ventricular myocardium in the Control group was uniform, with good activity and no abnormal beats. In the alcohol group, the activity of the left ventricular myocardium was weakened (Fig. 2B). HE staining results demonstrated that the myocardial cells in the alcohol group exhibited compensatory hypertrophy, no inflammatory cell infiltration; it also exhibited fibrosis, disordered arrangement of fibers, clear border between infarct and non-infarct border, a small amount of inflammatory cell infiltration can be seen (Fig. 2C). The success of model formation rate was $>80 \%$.

Enhanced LVDD with reduced EF and FS is demonstrated in rat models of ACM-amelioration of cardiac functions by valsartan. LVDD was increased with a decreased EF and FS in rat models of ACM (alcohol group) compared with the control group. The corresponding levels in the treatment group 
Table I. LVDD, EF and FS in every group.

\begin{tabular}{llllll}
\hline Groups & LVDD $(\mathrm{mm})$ & EF $(\%)$ & FS $(\%)$ & LVSD (mm) & E/A ratio \\
\hline Control $(\mathrm{n}=15)$ & $5.23 \pm 0.69$ & $73.45 \pm 8.35$ & $47.46 \pm 4.36$ & $2.89 \pm 0.65$ & $1.98 \pm 0.32$ \\
Alcohol (n=15) & $7.76 \pm 0.65^{\mathrm{a}}$ & $43.12 \pm 5.34^{\mathrm{a}}$ & $30.56 \pm 2.45^{\mathrm{a}}$ & $5.78 \pm 0.46$ & $0.94 \pm 0.25$ \\
Alcohol + valsartan $(\mathrm{n}=15)$ & $6.39 \pm 0.73^{\mathrm{a}, \mathrm{b}}$ & $54.34 \pm 5.38^{\mathrm{a}, \mathrm{b}}$ & $35.74 \pm 3.65^{\mathrm{a}, \mathrm{b}}$ & $4.15 \pm 0.39$ & $1.63 \pm 0.21$ \\
\hline
\end{tabular}

${ }^{\mathrm{a}} \mathrm{P}<0.05$ vs. the control group; ${ }^{\mathrm{b}} \mathrm{P}<0.05$ vs. the alcohol + valsartan group. LVDD, left ventricular end diastolic diameter; EF, ejection fraction; FS, fractional shortening; LVSD, left ventricular end systolic diameter; E/A ratio, early /atrial ratio.
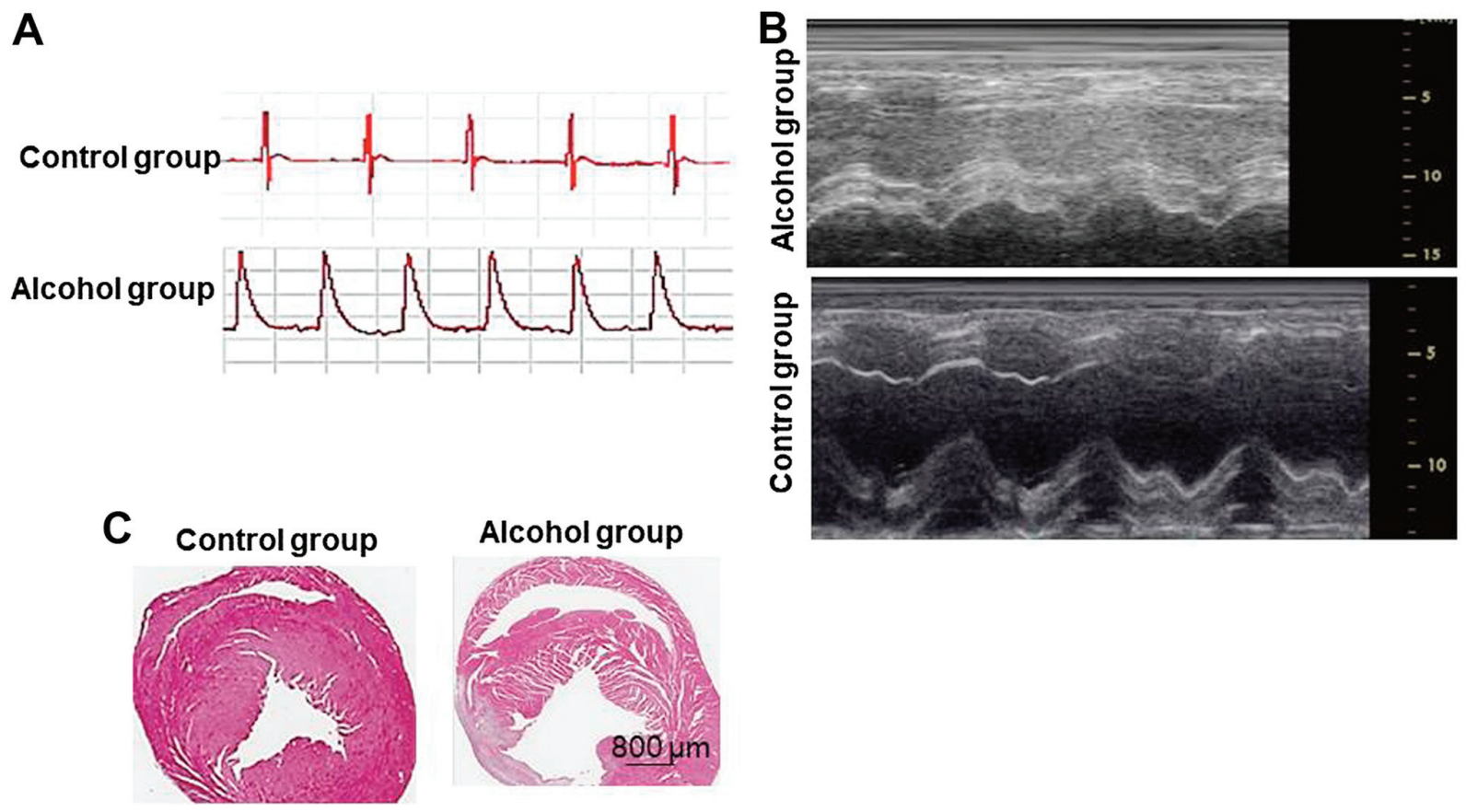

Figure 2. Verification of acute myocardial infarction model. (A) Electrocardiogram of the alcohol group and control group. (B) Ultrasound detection in the control group and the alcohol group. (C) Hematoxylin and eosin staining of in myocardial cells in the rat model of three groups (control group, alcohol group and alcohol + valsartan group) (original magnification, $\mathrm{x} 200$ ).

were between the control group and the alcohol group. The detected differences were statistically significant. The results of the present study suggest enlargement of the left ventricle with decreased EF and myocardial contractility associated with alcohol intake (Table I), which were ameliorated by the use of valsartan that led to improved cardiac function.

Disorganized arrangement and increased fibrosis of myocardial filaments in rat model of ACM-rectification by valsartan. In the alcohol group, HE staining revealed a disorganized arrangement and rupture of myocardial filaments, an enlarged intercellular space with edema and massive inflammatory infiltration indicated that cells were signaled to undergo apoptosis (Fig. 3A) compared with the control group, which displayed ordered arrangement of myocardial filaments, evenly distributed cytoplasm without rupture, enlargement of intercellular space, effusion edema or inflammatory infiltration. In the treatment group, cells were in a closely packed arrangement with reduced infiltration of inflammatory cells compared with the alcohol group. Masson's trichrome staining revealed increased fibrosis of myocardial cells in the alcohol group compared with the control group with no fibrosis (Fig. 3B). The degree of fibrosis in the treatment group was between the control and alcohol groups. The results of the present study indicate alleviation of enhanced fibrosis of myocardial cells in ACM by valsartan.

Elevated RhoA and ROCK in myocardial tissues of rat models of ACM-reversal by valsartan. In the myocardial tissue, IHC results demonstrated elevated expression of RhoA and ROCK in the alcohol group compared with the control group (Fig. 4A and 4B). The myocardial expression of RhoA and ROCK was decreased in the treatment group compared with in the alcohol group.

Augmented protein and $m R N A$ expressions of RhoA and ROCK and decreased MYL in myocardial cells-amelioration by valsartan. In the myocardial tissue, the expression of RhoA and ROCK were significantly elevated and MYL was significantly decreased in the alcohol group compared with the control group $(\mathrm{P}<0.05$; Fig. 5A-D). The myocardial expression of RhoA and ROCK were significantly downregulated along 
A

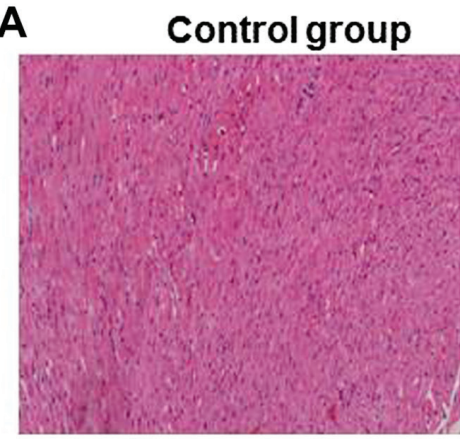

B Control group

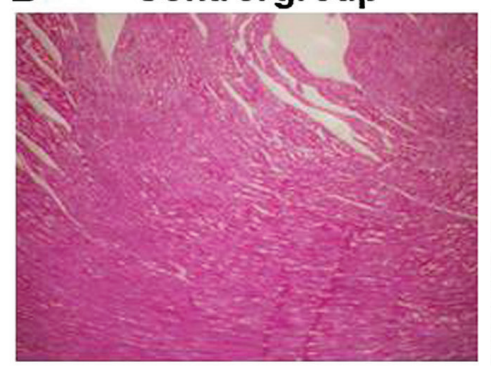

Alcohol group

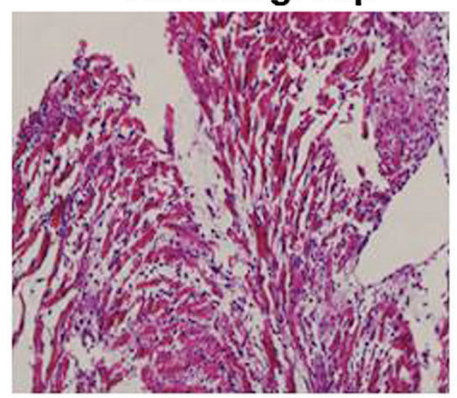

Alcohol group

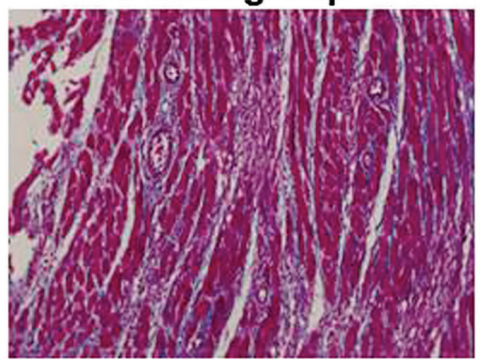

Alcohol+Vasartan group

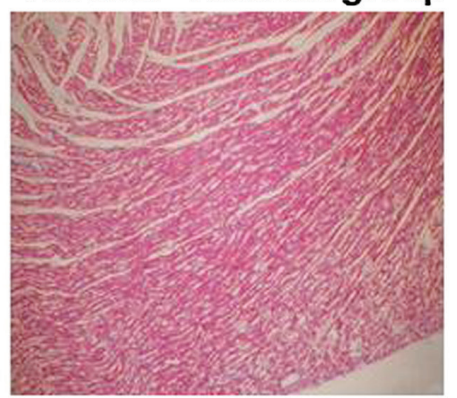

Alcohol+Vasartan group

Figure 3. Representative images of myocardial filaments disorganized arrangement and increased fibrosis in a rat model of alcoholic cardiomyopathy-rectification by valsartan. (A) Hematoxylin and eosin staining of myocardial filaments arrangement in a rat model of the three groups (control group, alcohol group and alcohol + valsartan group) (original magnification, x200). (B) Masson's trichrome staining of myocardial cells fibrosis in a rat model of the three groups (control group, alcohol group and alcohol + valsartan group) (original magnification, x200).

with upregulation of MYL in the treatment group compared with the alcohol group $(\mathrm{P}<0.05)$. In the myocardial tissue, fluorescence quantitative PCR results indicated elevated mRNA expressions of RhoA and ROCK in the alcohol group compared with the control group $(\mathrm{P}<0.05$; Fig. 5E and $\mathrm{F})$. The mRNA expressions of RhoA and ROCK were decreased in the treatment group compared with the alcohol group $(\mathrm{P}<0.05$; Fig. 5E and 5F). The mRNA expression of MYL was decreased in the alcohol group compared with the control group. The mRNA expression of MYL was increased in the treatment group compared with the alcohol group ( $\mathrm{P}<0.05$; Fig. $5 \mathrm{G})$.

\section{Discussion}

Alcohol abuse is injurious to health. Long-term or intermittent addiction to alcohol is accountable for gastrointestinal diseases, alcoholic liver diseases, myopathy and encephalopathy. The associated cardiovascular diseases demonstrate symptoms similar to dilated cardiomyopathy and are referred to as ACM $(23,24)$. Patients at the end-stage of ACM suffer from decreased cardiac function leading to heart failure or arrhythmia. Without any effective measures, including abstinence, nearly half of the patients would die in a period of 4 years $(25,26)$. However, the pathogenesis and treatment methods of ACM remain unclear. In the present study, the role of the variations in the RhoA-ROCK2-MYL pathway were investigated in the pathogenesis of $\mathrm{ACM}$ and the beneficial effects of angiotensin-converting enzyme inhibitor I drugs.

Ras, the first-identified low-molecular weight $\mathrm{G}$ protein (27) belongs to a family of proteins which is divided into three subgroups, namely, RhoA, RhoB and RhoC. RhoA is a major member involved in multiple intracellular signal transduction pathways (28-30). ROCK is a member of the Ser/Thr protein kinase family. It is the key and characteristic downstream signaling molecule of RhoA (31-33) consisting of ROCK1 and ROCK $2(34,35)$. ROCK1 is mainly expressed in the lung, liver, kidney, spleen and testicles, while ROCK2 is expressed in the heart and brain $(36,37)$. MYL is a major downstream protein of ROCK and together with the myosin heavy chain constitutes myosin $(29,38)$. MYL is a key substance in tubulin with major regulatory effects on the contraction of myocardial cells. Previous studies (39-41) have demonstrated that chronic alcohol intake activates the renin-angiotensin system and through Angiotensin II (AngII) facilitates cardiac remodeling. Blocking the angiotensin type 1 (AT1) receptor ameliorates cardiac remodeling.

A massive intake of alcohol activates RAS and facilitates binding of AngII to AT1 (42). This activates the downstream RhoA resulting in the induction of the expression of ROCK. Sequentially, ROCK inhibits its downstream protein MYL, therefore decreasing its expression. MYL is critical for the contraction of myocardial cells (43). Reduced expression of MYL is associated with decreased contraction of myocardial cells and as time lapses, patients become more susceptible to heart failure.

Through alcohol gavage and free access to alcohol, rat models of ACM were established. The method surmounted the limitations of having only free access to alcohol, individual differences in models and long time needed for model establishment. Pathological manifestations in the alcohol group included disorganized arrangement and rupture of myocardial filaments, an enlarged intercellular space with edema, and massive inflammatory infiltration which indicated that cells were signaled to undergo apoptosis the alcohol group. The treatment group demonstrated an ordered arrangement of myocardial filaments, evenly distributed cytoplasm without 
A Control group

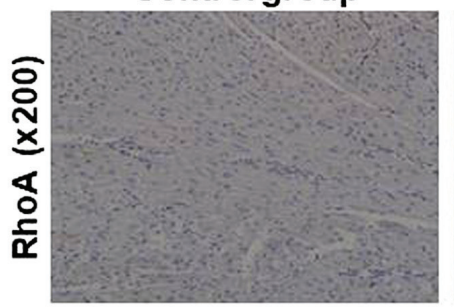

B

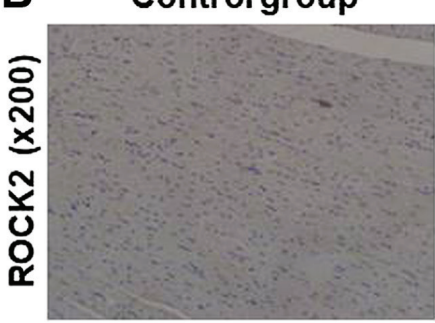

Alcohol group

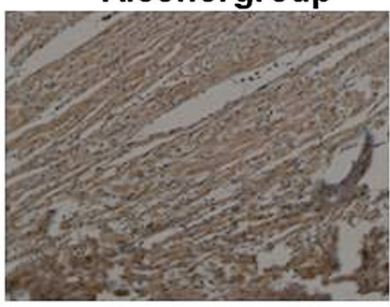

Alcohol group

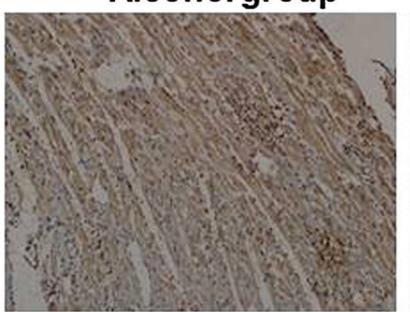

Alcohol+Vasartan group

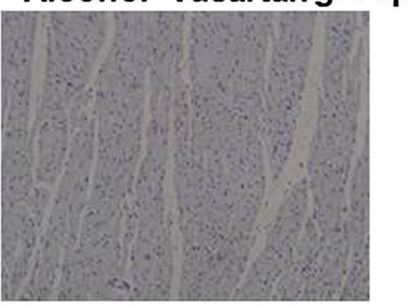

Alcohol+Vasartan group

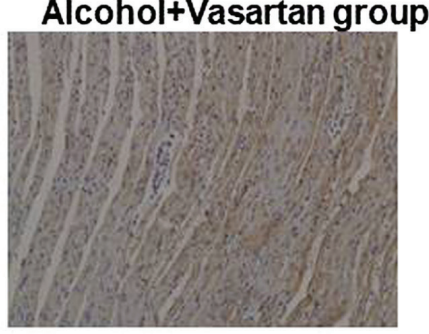

Figure 4. Elevated RhoA and ROCK in myocardial tissues of rat models of ACM-reversal by valsartan. Immunohistochemical staining of myocardial tissues of rat models for RhoA (A) and ROCK (B) expression. ACM group myocardial tissues were scored as high RhoA and ROCK expression of ACM and reversal by valsartan (original magnification, x200). ACM, alcoholic cardiomyopathy; ROCK, Rho-associated protein kinase; RhoA, Ras homolog gene family, member A.

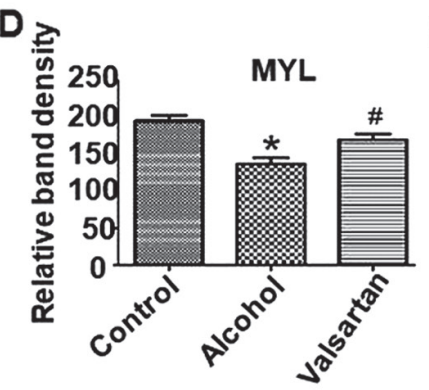

A

B

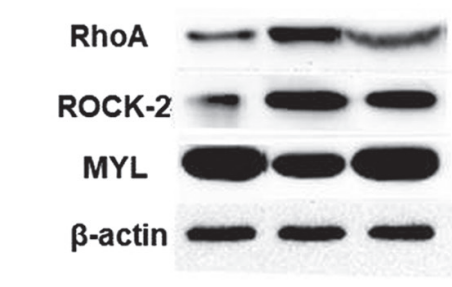

B

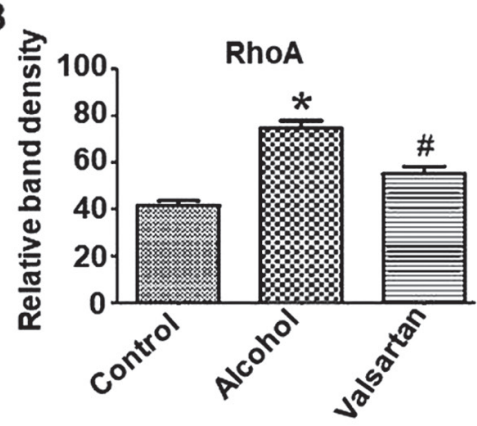

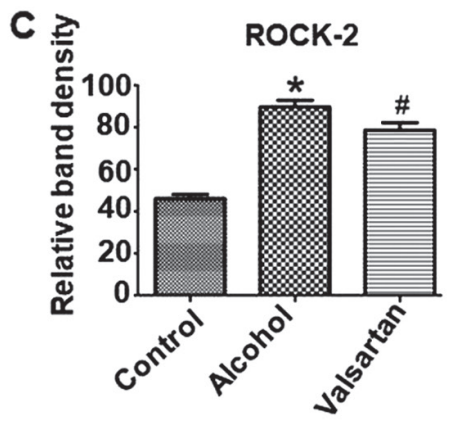
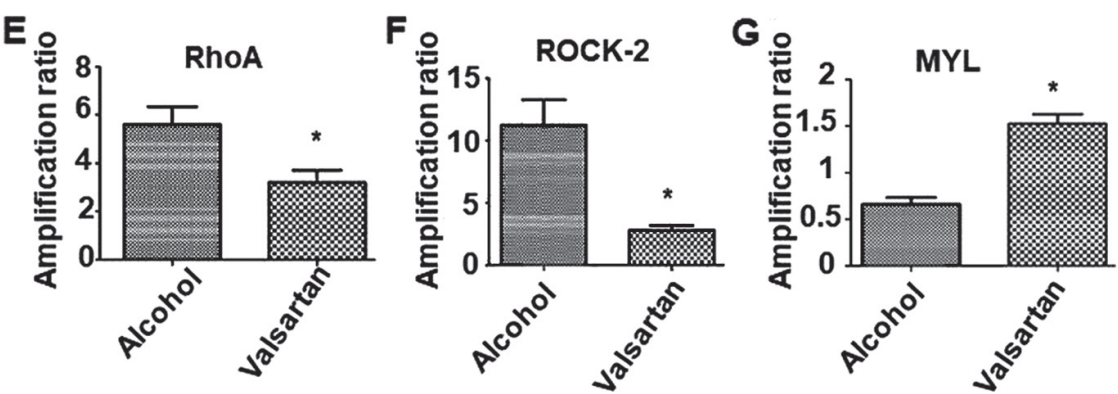

Figure 5. Effect of valsartan on protein expression level of RhoA, ROCK-2 and MYL in myocardial cells-amelioration by valsartan. (A) Western blots results of RhoA, ROCK-2 and MYL expression in myocardial cells. The experiment was repeated 3 times with similar results. The figure presents a representative analysis. Quantification of the western blots (B) RhoA, (C) ROCK-2 (D) and MYL. Columns are means $+/-$ standard error of the mean, ( $\mathrm{n}=3$ ). ${ }^{*} \mathrm{P}<0.05$, alcohol group compared with the control group. ${ }^{\#} \mathrm{P}<0.05$, alcohol + valsartan group compared with the alcohol group. Reverse transcription-quantitative polymerase chain reaction analysis of the (E) RhoA, (F) ROCK-2 and (G) MYL mRNA transcription profiles of myocardial cells in the alcohol group and alcohol + vasartan group $(n=3)$. Expression levels were normalized to GAPDH levels. * $\mathrm{P}<0.05$, alcohol + valsartan group compared with the alcohol group. ROCK, Rho-associated protein kinase; RhoA, Ras homolog gene family, member A; MYL, myosin light chain.

any rupture and absence of enlarged intercellular space, effusion edema or inflammatory infiltration. Results of Masson's trichrome staining demonstrated significantly enhanced fibrosis of myocardial cells in the alcohol group compared with the control group. Myocardial fibrosis was not observed in the treatment group suggesting that valsartan alleviated myocardial fibrosis leading to amelioration of cardiac remodeling in ACM. Echocardiography revealed decreased EF and increased LVEDD in the treatment group, suggesting a decline in systolic function. The treatment group demonstrated decreased LVEDD and increased EF compared with the alcohol group, with a concomitant increase in the contractility of myocardial cells. These results suggest that long-term massive intake of alcohol initiates myocardial injury, fibrosis and systolic dysfunction, leading to decreased cardiac function, or heart failure. Valsartan improves cardiac function by preventing the progression of ACM into heart failure, thereby benefiting majority of the patients. 
IHC was employed to detect the expressions of RhoA and ROCK in the myocardial cells (44), while western blotting was used to detect the expressions of RhoA, ROCK and MYL. Results demonstrated that in comparison with the control group, western blot analysis detected elevated expression of RhoA and ROCK and decreased expression of MYL in the alcohol group compared with the control group. Decreased RhoA and ROCK, and elevated MYL expression was detected in the treatment group compared with the alcohol group. PCR analysis revealed increased RhoA and ROCK and downregulated MYL mRNA expressions in the alcohol group compared with the control group. Notably, decreased RhoA and ROCK and elevated MYL mRNA expression was seen in the treatment group compared with in the alcohol group, which was consistent with the results of western blotting. These findings suggest the activation of the RhoA-ROCK2-MYL pathway at the protein or mRNA level by alcohol and its metabolites causing a reduction in myocardial contractility. Treatment with valsartan inhibited AT1 and suppressed the expression of RhoA and ROCK to increase the expression of MYL, thereby enhancing the contractility of myocardial cells. Valsartan improves cardiac function and exerts a therapeutic and prophylactic effect in ACM. Early administration of angiotensin receptor antagonists ameliorates cardiac function and the prognosis of ACM, therefore indicating its critical protective effect in the development and progression of ACM. A rat model of ACM revealed increased LVEDD, decreased EF and systolic function, and cell rupture associated with increased fibrosis (45). Administration of valsartan ameliorated myocardial fibrosis and prevented the progression of ACM. Alcoholic stimulation activates the RhoA-ROCK2-MYL pathway to curb the systolic function of myocardial cells (46), while valsartan inhibits this pathway to enhance myocardial contractility and improves cardiac function.

Although alcohol gavage + free access to alcohol can shorten the time required to simulate and establish the development of ACM more effectively, success rate remains quite low due to incompetence in gavaging, excessively high concentrations of alcohol, and treatment with industrial alcohol instead of the wine made from grain. In addition, due to the lack of RhoA and ROCK inhibitors, the involvement of the RhoA-ROCK2-MYL pathway was not confirmed. The results of the present study are inconsistent with other research results that ROCK2 leads to the activation of myosin light chain by phosphorylation (47). It was speculated that MYL phosphorylation is determined by the balance between the activities of Rho-kinase and myosin phosphatase. Also, apart from ROCK2, an additional pathway(s) may be required for sustained MYL phosphorylation.

Experimental design determining the drug concentration was simple and further evaluation on dose-effect association was not performed. Because of the lack of abstinence group or abstinence + valsartan group, the prophylactic effect of valsartan could only be proved in ACM. Therefore, further studies are necessary for validation of the therapeutic effects of valsartan.

\section{Acknowledgements}

Not applicable.

\section{Funding}

No funding was received.

\section{Availability of data and material}

The datasets used and/or analyzed during the current study are available from the corresponding author on reasonable request.

\section{Authors' contributions}

In this study, LL and WL conceived the study and designed the experiments. $\mathrm{LJ}$ and $\mathrm{JZ}$ contributed to the data collection, $\mathrm{JL}$ and WY performed the data analysis and interpreted the results. LL wrote the manuscript; WL and LZ contributed to the critical revision of article. All authors read and approved the final manuscript.

\section{Ethics approval and consent to participate}

This study was approved by the First Affiliated Hospital of Harbin Medical University.

\section{Patient consent to participate}

Not applicable.

\section{Competing interests}

The authors declare that they have no competing interests.

\section{References}

1. Piano MR: Alcoholic cardiomyopathy: Incidence, clinical characteristics, and pathophysiology. Chest 121: 1638-1650, 2002.

2. Skotzko CE, Vrinceanu A, Krueger L and Freudenberger R: Alcohol use and congestive heart failure: Incidence, importance, and approaches to improved history taking. Heart Fail Rev 14: 51-55, 2009.

3. Berger $\mathbf{J}$ and Moller DE: The mechanisms of action of PPARs. Annu Rev Med 53: 409-435, 2002.

4. Finck BN, Han X, Courtois M, Aimond F, Nerbonne JM, Kovacs A, Gross RW and Kelly DP: A critical role for PPARalpha-mediated lipotoxicity in the pathogenesis of diabetic cardiomyopathy: Modulation by dietary fat content. Proc Natl Acad Sci USA 100: 1226-1231, 2003.

5. Barger PM, Brandt JM, Leone TC, Weinheimer CJ and Kelly DP: Deactivation of peroxisome proliferator-activated receptor-alpha during cardiac hypertrophic growth. J Clin Invest 105: 1723-1730, 2000.

6. Berger J and Moller DE: The mechanisms of action of PPARs. Annu Rev Med 53: 409-435, 2002.

7. Mishra PK, Tyagi N, Kundu S and Tyagi SC: MicroRNAs are involved in homocysteine-induced cardiac remodeling. Cell Biochem Biophys 55: 153-162, 2009.

8. Hultberg B, Berglund M, Andersson A and Frank A: Elevated plasma homocysteine in alcoholics. Alcohol Clin Exp Res 17: 687-689, 1993.

9. Stickel F, Choi SW, Kim YI, Bagley PJ, Seitz HK, Russell RM, Selhub J and Mason JB: Effect of chronic alcohol consumption on total plasma homocysteine level in rats. Alcohol Clin Exp Res 24: 259-264, 2000.

10. Dettmeyer R, Reith K and Madea B: Alcoholic cardiomyopathy versus chronic myocarditis-immunohistological investigations with LCA, CD3, CD68 and tenascin. Forensic Sci Int 126: 57-62, 2002.

11. Yang QF and Li YH: Roles of PPARs on regulating myocardial energy and lipid homeostasis. J Mol Med (Berl) 85: 697-706, 2007. 
12. Pellieux C, Montessuit C, Papageorgiou I and Lerch R: Inactivation of peroxisome proliferator-activated receptor isoforms alpha, beta/delta, and gamma mediate distinct facets of hypertrophic transformation of adult cardiac myocytes. Pflugers Arch 455: 443-454, 2007.

13. Loichot C, Jesel L, Tesse A, Tabernero A, Schoonjans K, Roul G, Carpusca I, Auwerx J and Andriantsitohaina R: Deletion of peroxisome proliferator-activated receptor-alpha induces an alteration of cardiac functions. Am J Physiol Heart Circ Physiol 291: H161-H166, 2006.

14. Melendez J, Welch S, Schaefer E, Moravec CS, Avraham S Avraham H and Sussman MA: Activation of pyk2/related focal adhesion tyrosine kinase and focal adhesion kinase in cardiac remodeling. J Biol Chem 277: 45203-45210, 2002.

15. Preedy VR, Patel VB, Reilly ME, Richardson PJ, Falkous G and Mantle D: Oxidants, antioxidants and alcohol: Implications for skeletal and cardiac muscle. Front Biosci 4: e58-e66, 1999.

16. Elamin E, Masclee A, Dekker J and Jonkers D: Ethanol disrupts intestinal epithelial tight junction integrity through intracellular calcium-mediated Rho/ROCK activation. Am J Physio Gastrointest Liver Physiol 306: G677-G685, 2014.

17. Wei B, Shang YX, Li M, Jiang J and Zhang H: Cytoskeleton changes of airway smooth muscle cells in juvenile rats with airway remodeling in asthma and the RhoA/ROCK signaling pathway mechanism. Genet Mol Res 13: 559-569, 2014.

18. Li Z, Liang J, Wu WK, Yu X, Yu J, Weng X and Shen J: Leptin activates RhoA/ROCK pathway to induce cytoskeleton remodeling in nucleus pulposus cells. Int J Mol Sci 15: 1176-1188, 2014

19. Shimokawa $\mathrm{H}$ and Satoh K: Light and dark of reactive oxygen species for vascular function: 2014 ASVB (Asian Society of Vascular Biology). J Cardiovasc Pharmacol 65: 412-418, 2015.

20. Satoh K, Godo S, Saito H, Enkhjargal B and Shimokawa H: Dual roles of vascular-derived reactive oxygen species-with a special reference to hydrogen peroxide and cyclophilin A. J Mol Cell Cardiol 73: 50-56, 2014

21. Higashi M, Shimokawa H, Hattori T, Hiroki J, Mukai Y, Morikawa K, Ichiki T, Takahashi S and Takeshita A: Long-term inhibition of Rho-kinase suppresses angiotensin II-induced cardiovascular hypertrophy in rats in vivo: Effect on endothelial NAD(P)H oxidase system. Circ Res 93: 767-775, 2003.

22. Livak KJ and Schmittgen TD: Analysis of relative gene expression data using real-time quantitative PCR and the 2(T)(-Delta Delta C) method. Methods 25: 402-408, 2001

23. Fiarresga A, Cacela D, Galrinho A, Ramos R, de Sousa L, Bernardes L, Patrício L and Cruz Ferreira R: Alcohol septal ablation in obstructive hypertrophic cardiomyopathy: Four years of experience at a reference center. Rev Port Cardiol 33: 1-10, 2014.

24. Veselka J, Lawrenz T, Stellbrink C, Zemanek D, Branny M, Januska J, Sitar J, Dimitrow P, Krejci J, Dabrowski M, et al: Early outcomes of alcohol septal ablation for hypertrophic obstructive cardiomyopathy: A European multicenter and multinational study. Catheter Cardiovasc Interv 84: 101-107, 2014.

25. Pridemore WA, Chamlin MB, Kaylen MT and Andreev E: The effects of the 2006 Russian alcohol policy on alcohol-related mortality: An interrupted time series analysis. Alcohol Clin Exp Res 38: 257-266, 2014.

26. Kycina P and Murin J: Alcoholic cardiomyopathy and cardiovascular events-an insight from the Liptov region. Bratisl Med J 114: 337-341, 2013

27. Lessey-Morillon EC, Osborne LD, Monaghan-Benson E, Guilluy C, O'Brien ET, Superfine R and Burridge K: The RhoA guanine nucleotide exchange factor, LARG, mediates ICAM-1-dependent mechanotransduction in endothelial cells to stimulate transendothelial migration. J Immunol 192: 3390-3398, 2014.

28. Duan X, Liu J, Dai XX, Liu HL, Cui XS, Kim NH, Wang ZB, Wang Q and Sun SC: Rho-GTPase effector ROCK phosphorylates cofilin in actin-meditated cytokinesis during mouse oocyte meiosis. Biol Reprod 90: 37, 2014.

29. Gabrielli L, Winter JL, Godoy I, McNab P, Padilla I, Cordova S Rigotti P, Novoa U, Mora I, García L, Ocaranza MP and Jalil JE: Increased rho-kinase activity in hypertensive patients with left ventricular hypertrophy. Am J Hypertens 27: 838-845, 2014.

30. Hensel N, Stockbrügger I, Rademacher S, Broughton N, Brinkmann H, Grothe C and Claus P: Bilateral crosstalk of rho- and extracellular-signal-regulated-kinase (ERK) pathways is confined to an unidirectional mode in spinal muscular atrophy (SMA). Cell Signal 26: 540-548, 2014.
31. Morgan-Fisher M, Couchman JR and Yoneda A: Phosphorylation and mRNA splicing of collapsin response mediator protein-2 determine inhibition of rho-associated protein kinase (ROCK) II function in carcinoma cell migration and invasion. J Biol Chem 288: 31229-31240, 2013.

32. Sasaki T, Oga T, Nakagaki K, Sakai K, Sumida K, Hoshino K, Miyawaki I, Saito K, Suto F and Ichinohe N: Developmental genetic profiles of glutamate receptor system, neuromodulator system, protector of normal tissue and mitochondria, and reelin in marmoset cortex: Potential molecular mechanisms of pruning phase of spines in primate synaptic formation process during the end of infancy and prepuberty (II). Biochem Biophys Res Commun 444: 307-310, 2014

33. Gaio V, Nunes B, Fernandes A, Mendonça F, Horta Correia F, Beleza A, Gil AP, Bourbon M, Vicente A, Dias CM and Barreto da Silva M: Genetic variation at the CYP2C19 gene associated with metabolic syndrome susceptibility in a South Portuguese population: Results from the pilot study of the European health examination survey in portugal. Diabetol Metab Syndr 6: 23, 2014.

34. Liu PY and Liao JK: A method for measuring Rho kinase activity in tissues and cells. Methods Enzymol 439: 181-189, 2008.

35. Mertsch S and Thanos S: Opposing signaling of ROCK1 and ROCK 2 determines the switching of substrate specificity and the mode of migration of glioblastoma cells. Mol Neurobiol 49: 900-915, 2014

36. Shimizu T, Fukumoto Y, Tanaka S, Satoh K, Ikeda S and Shimokawa H: Crucial role of ROCK2 in vascular smooth muscle cells for hypoxia-induced pulmonary hypertension in mice. Arterioscler Thromb Vasc Biol 33: 2780-2791, 2013.

37. Laeno AM, Tamashiro DA and Alarcon VB: Rho-associated kinase activity is required for proper morphogenesis of the inner cell mass in the mouse blastocyst. Biol Reprod 89: 122, 2013.

38. Al-Shboul O: The role of the RhoA/ROCK pathway in gender-dependent differences in gastric smooth muscle contraction. J Physiol Sci 66: 85-92, 2016.

39. Nzegwu MA, Okafor OC, Olusina DB, Ike V and Mbah AU: Dilated alcoholic cardiomyopathy and incidental lymphoma occuring in a 56 year old man who was being managed for hypertensive heart disease in Enugu Nigeria-a rare finding. Niger J Med 20: 494-497, 2011.

40. Jing L, Zhou LJ, Zhang FM, Li WM and Sang Y: Tenascin-x facilitates myocardial fibrosis and cardiac remodeling through transforming growth factor- $\beta 1$ and peroxisome proliferator-activated receptor $\gamma$ in alcoholic cardiomyopathy. Chin Med J (Engl) 124: 390-395, 2011

41. Tan Y, Li XK, Prabhu SD, Brittian KR, Chen Q, Yin X, McClain CJ, Zhou Z and Cai L: Angiotensin II plays a critical role in alcohol-induced cardiac nitrative damage, cell death, remodeling, and cardiomyopathy in a protein kinase $\mathrm{C} /$ nicotinamide adenine dinucleotide phosphate oxidase-dependent manner. J Am Coll Cardiol 59: 1477-1486, 2012.

42. Ferrario CM: Cardiac remodelling and RAS inhibition. Ther Adv Cardiovasc Dis 10: 162-171, 2016.

43. McArthur L, Chilton L, Smith GL and Nicklin SA: Electrical consequences of cardiac myocyte: Fibroblast coupling. Biochem Soc Trans 43: 513-518, 2015.

44. Chen Z, Liu S, Xia Y and Wu K: MiR-31 regulates Rho-associated kinase-myosin light chain (ROCK-MLC) pathway and inhibits gastric cancer invasion: Roles of RhoA. Med Sci Monit 22: 4679-4691, 2016.

45. Sabater-Molina M, Pérez-Sánchez I, Hernández Del Rincón JP and Gimeno JR: Genetics of hypertrophic cardiomyopathy: A review of current state. Clin Genet 93: 3-14, 2018.

46. Lopez NC, Ebensperger G, Herrera EA, Reyes RV, Calaf G, Cabello G, Moraga FA, Beñaldo FA, Diaz M, Parer JT and Llanos AJ: Role of the RhoA/ROCK pathway in high-altitude associated neonatal pulmonary hypertension in lambs. Am J Physiol Regul Integr Comp Physiol 310: R1053-R1063, 2016.

47. Bhadriraju K, Yang M, Alom Ruiz S, Pirone D, Tan J and Chen CS: Activation of ROCK by RhoA is regulated by cell adhesion, shape, and cytoskeletal tension. Exp Cell Res 313: 3616-3623, 2007.

This work is licensed under a Creative Commons Attribution-NonCommercial-NoDerivatives 4.0 International (CC BY-NC-ND 4.0) License. 